\title{
Decision Support Tool for Prediction of Critical Data to the Satellite Integrity
}

\author{
Primavera Botelho de Souza ${ }^{1}$, Mauricio Gonçalves Vieira Ferreira ${ }^{2}$, José Demisio Simões da Silva ${ }^{3}$, \\ and Ana Maria Ambrosio. \\ ${ }^{1}$ Applied Computing Postgraduate Program (CAP) - National Institute for Space \\ Research, São José dos Campos, SP, 12227-010, Brazil. \\ ${ }^{2}$ Satellite Control and Tracking Center (CRC) - National Institute for Space Research, \\ São José dos Campos, SP, 12227-010, Brazil. \\ ${ }^{3}$ Applied Mathematics and Computing Associated Laboratory (LAC) - National \\ Institute for Space Research, São José dos Campos, SP, 12227-010, Brazil. \\ ${ }^{4}$ Ground System Department (DSS) - National Institute for Space Research, São José \\ dos Campos, SP, 12227-010, Brazil. \\ prima@laser.inpe.br, mauricio@ccs.inpe.br, demisio@lac.inpe.br, \\ ana@dss.inpe.br
}

\begin{abstract}
The perspectives of multiple launchings, in the near future, by INPE's satellite program motivated the development of an application using temporal planning techniques based on Artificial Intelligence (AI) concepts. It will be used for automatic generation of flight operation plans to control satellite activities. However, making a critical analysis of these plans before real execution is impossible. We proposed a different approach using a decision support tool combining Bayesian Networking and AI-based data mining techniques for data prediction, aiming to maintain the integrity of the satellite.
\end{abstract}

\section{Introduction}

There is general interest in automating satellite control operations related to the task of controlling multiple satellites in INPE's Space Program. In addition, it is generally accepted that the automation of satellite control activities represents a way of reducing in-orbit satellite maintenance costs. At INPE, autonomous systems to control satellite operations employing Artificial Intelligence are being developed to automate ground segment operations.

However, this increased autonomy in satellite control operations can lead to distrust of the automatic control system behavior as compared to that of the well known and routine manual control system. In such cases, these systems still require an improvement in reliability to become operational.

In order to achieve this breakthrough in reliability, predictability and safety, an AIbased strategy for automatic validation of a flight operations plan generated by a planner is presented. This is an architecture composed of software components, resulting from the combination of verification and validation techniques. As a relevant part of this strategy, a decision support tool is proposed in this article, to assist experts 
in evaluating the actions of the plan, aiming at guaranteeing the integrity of the satellite. This tool consists of software using Artificial Intelligence techniques aimed at predicting the behavior of critical platform subsystems, such as the power supply subsystem, directly affected by the actions contained in each flight operation plan.

This paper presents in the following section some concepts related to the automation of the control activities of the satellite in orbit. Section 3 describes the strategy for validation of a flight operations plan, an overview of the relevant architecture and general behavior. Section 4 discusses some Artificial Intelligence techniques for data prediction, such as Bayesian methods which make the required learning a form of probabilistic inference, and data mining. Section 5 presents the design of the tool being developed for prediction. Conclusions are presented in Section 6.

\section{Satellite Flight Operation Plan}

The Flight Operations Plan (POV) includes the planning of control operations of space missions and ground segment activities for the planning, execution and control of the satellite in orbit. Each $P O V$ aims to maintain the satellite in orbit, working to achieve the goals of the mission, containing all the necessary information to control the satellite in orbit, such as: procedures for flight control, procedures for recovery of contingencies, rules, plans and schedules. All activities included in a $P O V$ have as their starting point the passage of the satellite over the Earth station. The amount of time that a satellite is visible to a given Earth station determines the set of flight operations that should be performed during each pass. Among the activities to control for this period is the sending of commands from the ground (telecommand), and the reception of telemetry which indicates the general state of the satellite.

To meet the growing demand for satellites in orbit and reduce costs significantly, recent studies in AI-based planning have been aimed at the development of tools that automate the tasks of controlling ground operations in INPE. The system called Intelligent Planning of Flight Operation Plans (PlanIPOV) [Cardoso et al. 2006], uses temporal planning AI techniques (temporal planner) applied to the automatic generation of flight operation plans to support the activities of controlling satellites in orbit.

At the same time, the use of automatically generated POVs leads to many doubts. These are partly related to the new technologies involved, but the greatest resistance is related to reliability in the execution of these actions, the predictability and safety of satellites. This increase in autonomy can lead to suspicion about the behavior, often well known and routine. The set of actions contained in a plan acts directly on data critical to maintenance of the satellite integrity. Furthermore, depending on the demand for satellites in orbit, a careful validation of these plans can become unviable.

\section{Strategy for Validation of Flight Operations Plan}

The strategy of validation consist of an architecture composed of several software components for validation of a flight operations plan, generated automatically, to be executed in simulation before actual execution (Figure 1). It is designed on the basis of appropriate assurance techniques for space systems [Blanquart et al. 2004]. 


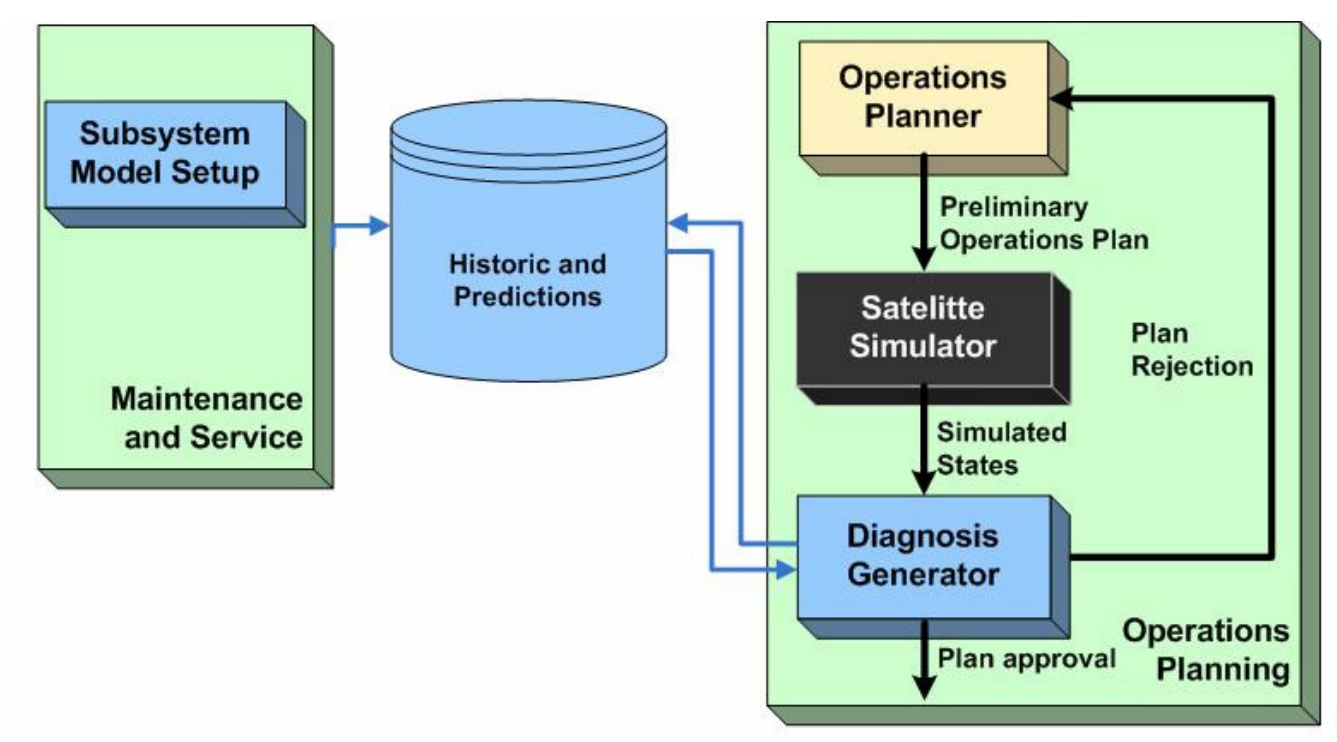

Figure 1. Validation of Flight Operations Plan: architecture and situation.

Through an execution off-line of the generated plan by the planner, each action of the plan is executed and a simulation of the behavior of the satellite is performed by a satellite simulator. The simulator is based on a virtual satellite, with simplified models, which is also part of the strategy for validation of the generated plan [Tominaga et al. 2008].

The simulator returns to a tool called the Diagnosis Generator, parameters and telemetries (see section 2) containing the simulated state of the satellite, resulting from the execution of the plan's actions. Upon receiving the status of the system, the Diagnosis Generator tool provides data on history and prediction for these parameters and telemetries, indicating how the general state of the satellite will evolve, suggesting the adoption or rejection of the plan.

\section{Techniques for Data Prediction}

Computational prediction models are based on probabilistic reasoning over time, interpreting the present and understanding the past and future forecast [Russel and Norvig 2004]. Prediction is one of the basic inference tasks in time models, in which the posterior distribution on the future state is calculated, given all the evidence to date. In short, predictive inference is an interpretation of probability that emphasizes the prediction of future behavior on the basis of past observations.

Bayesian methods make learning a form of probabilistic inference, using observations to update a distribution on the basis of a priori hypotheses. A Bayesian network, belief network or directed acyclic graphical model is a probabilistic graphical model that represents a set of random variables and their conditional independencies via a directed acyclic graph (DAG). Formally, Bayesian networks are directed acyclic graphs whose nodes represent variables, and whose missing edges encode conditional independencies between the variables. Nodes represent random variables, but in the Bayesian sense: they may be observable quantities, latent variables, unknown parameters or hypotheses. Efficient algorithms exist that perform inference and learning in Bayesian networks [Niedermayer 1998]. 
Data mining is another method, in which the ultimate goal is prediction, and represents a process developed to examine large amounts of data routinely collected. The term also refers to a collection of tools used to perform the process. It is an analytic process designed to explore data (usually large amounts of data) in search of consistent patterns and/or systematic relationships between variables, and then to validate the findings by applying the detected patterns to new subsets of data. The process of data mining consists of three stages: initial exploration, model building or pattern identification with validation/verification, and deployment (i.e., the application of the model to new data in order to generate predictions) [Fayyad et al. 1996]. Predictive data mining is used in most areas where data are collected - marketing, health, science, communications, etc.

\section{Diagnosis Generator: A Tool for Data Prediction}

As mentioned in section 3, the Diagnosis Generator tool should be able to provide results on history and data prediction for a critical subsystem. As an example, a set of telemetries, parameters and operational limits of a simplified model of a Power Supply Subsystem (PSS), based on a virtual satellite is used as input data (Table 1).

Table 1. Example of critical telemetry indicating the voltage values of a battery.

\begin{tabular}{|l|l|l|}
\hline TMD014 & TMD014 & TMD014 \\
\hline BAT1 VOLT & Min. BAT1 VOLT & Max. BAT1 VOLT \\
\hline VOLT & VOLT & VOLT \\
\hline 45,4 & 44,6 & 46 \\
\hline 45,9 & 43,2 & 49,1 \\
\hline 50 & 49,3 & 50,5 \\
\hline 51,1 & 50,6 & 51,6 \\
\hline 52,1 & 51,7 & 52,6 \\
\hline 53,2 & 52,7 & 53,8 \\
\hline 54,3 & 53,7 & 54,8 \\
\hline 54,3 & 53,6 & 54,9 \\
\hline 54,2 & 53,3 & 55 \\
\hline 54,2 & 53,8 & 54,9 \\
\hline 54,4 & 53,7 & 54,8 \\
\hline 50,9 & 49,6 & 54,4 \\
\hline 48,7 & 48 & 49,3 \\
\hline 47,2 & 46,7 & 47,8 \\
\hline 46 & 45,4 & 46,5 \\
\hline 44,7 & 43,1 & 46,9 \\
\hline 49,3 & 48,1 & 50,1 \\
\hline & &
\end{tabular}

The idea is to apply the two different techniques described in section 4 to these data (Table 1) to generate predictions about future satellite behavior, evaluating predictive results from each. For this, we plan to work in the following direction: the initial step is the construction of a prototype for analysis of the techniques applied for prediction. For implementation of techniques we employ the Waikato Environment for Knowledge Analysis (WEKA), a suite of machine learning software written in Java [University of Waikato 2006]. WEKA is free software available under the GNU General Public License, aiming to add algorithms from different approaches in the sub-area of Artificial Intelligence, dedicated to the study of learning by machines. 


\section{Conclusion}

This paper presented the design of a prediction tool being developed as a relevant part of the validation strategy for a flight operations plan generated automatically to control and track satellites. The tool applies Artificial Intelligence techniques for data prediction of critical platform subsystem, directly affected by the actions contained in each satellite flight plan. In addition, the tool assists experts in impact analysis of each plan's action on the satellite behavior, suggesting the adoption or rejection of the plan.

After concluding this implementation phase, the next step is to compare the results provided by each technique used for prediction with real data. With this prototype at hand, we will implement the Diagnosis Generator, to be integrated with the simulator, providing effective support to experts, and representing an advance in reliability, predictability and safety of the satellite control activities generated automatically, especially considering multiple launchings planned for the near future, when a careful evaluation of these plans, before real execution would be impossible.

\section{References}

Blanquart, P., Fleury, S., Hernek, M., Honvault, C., Ingrand, F., Poncet, J. C., Powell, D., Strady-Lécubin, N., and Thévenod-Fosse, P. (2004), Software Safety Supervision On-board Autonomous Spacecraft. In 2nd European Congress on Embedded Real Time Software (ERTS-2), Toulouse, France, 11p.

Cardoso, L. S., Ferreira, M. G. V, and Orlando, V. (2006), An Intelligent System for Generation of Automatic Flight Operation Plans for the Satellite Control Activities at INPE. In Proceedings of the 9th International Conference on Space Operations, Rome, Italy, June.

Fayyad, U., Piatetsky-Shapiro, G., Smyth, P. (1996). From data mining to knowledge discovery: An overview. In: Advances in Knowledge Discovery and Data Mining, AAAI Press, MIT, Cambridge, Massachusetts , p.1-34.

Niedermayer, D. (1998), An Introduction to Bayesian Networks and their Contemporary Applications, College of the North Atlantic-Qatar, Doha, Qatar, December.

Russell, S. and Norvig, P. (2004), Inteligência Artificial, Tradução da 2a. Edição, Editora Campus, Brazil.

Tominaga, J., Silva, J. D. S., Ferreira, M. G. V., (2008), A Proposal for Implementing Automation in Satellite Control Planning. International Committee on Technical Interchange for Space Mission Operations and Ground Data Systems (SpaceOps) Conference 2008, AIAA, Heidelberg, Germany, May 12-16.

University of Waikato (2006) Waikato Environment for Knowledge Analysis (WEKA), http://www.cs.waikato.ac.nz/ml/weka/, junho. 